\title{
On the role of electroweak bremsstrahlung for indirect dark matter signatures
}

\author{
M. Kachelrieß, ${ }^{1}$ P. D. Serpico, ${ }^{2,3}$ and M. Aa. Solberg ${ }^{1}$ \\ ${ }^{1}$ Institutt for fysikk, NTNU, N-7491 Trondheim, Norway \\ ${ }^{2}$ Physics Department, Theory Division, CERN, CH-1211 Geneva 23, Switzerland \\ ${ }^{3}$ LAPTH, UMR 5108, 9 chemin de Bellevue - BP 110, 74941 Annecy-Le-Vieux, France
}

(Dated: October 30, 2009)

\begin{abstract}
Interpretations of indirect searches for dark matter (DM) require theoretical predictions for the annihilation or decay rates of DM into stable particles of the standard model. These predictions include usually only final states accessible as lowest order tree-level processes, with electromagnetic bremsstrahlung and the loop-suppressed two gamma-ray line as exceptions. We show that this restriction may lead to severely biased results for DM tailored to produce only leptons in final states and with mass in the $\mathrm{TeV}$ range. For such models, unavoidable electroweak bremsstrahlung of $Z$ and $W$-bosons has a significant influence both on the branching ratio and the spectral shape of the final state particles. We work out the consequences for two situations: Firstly, the idealized case where DM annihilates at tree level with $100 \%$ branching ratio into neutrinos. For a given cross section, this leads eventually to "minimal yields" of photons, electrons, positrons and antiprotons. Secondly, the case where the only allowed two-body final states are electrons. The latter case is typical of models aimed at fitting cosmic ray $e^{-}$and $e^{+}$data. We find that the multimessenger signatures of such models can be significantly modified with respect to results presented in the literature.
\end{abstract}

PACS numbers: 95.35.+d, 95.85.Pw, 98.70.Sa

\section{INTRODUCTION}

Despite the numerous cosmological and astrophysical indications for the presence of nonbaryonic dark matter (DM), the particle nature of DM has yet to be identified. One strategy towards DM "detection" is to search for its self-annihilation (or decay) products in our Galaxy, provided that the annihilation cross section $\langle\sigma v\rangle$ (or the decay rate) is large enough. Restricting the space of candidates to weakly interacting massive thermal relics, $\langle\sigma v\rangle$ (at freeze-out) is fixed by the DM abundance while the DM mass $m_{X}$ lies in this class of models typically within one or two orders of magnitude off the weak scale $m_{W}$. Nevertheless, considerable model-dependence remains because of our ignorance of the final states produced in the annihilation process, which vary from model to model.

Under the sole hypothesis that massive dark matter annihilates into standard model particles, a few years ago an interesting conservative upper bound on $\langle\sigma v\rangle$ was derived using data on the least detectable final states, namely neutrinos $[1,2]$. (Similar considerations apply to decaying particles [3], although we will not mention this further.) It is natural to ask if these conservative bounds can be improved or consolidated further. It was shown previously in Ref. [4] that electroweak bremsstrahlung leads to a break-down of perturbation theory and a nonnegligible branching ratio in electromagnetic channels for $m_{X} \gg m_{W}$, even if at tree level DM couples only neutrinos. This argument was used then in Refs. $[5,6]$ to derive constraints on the DM annihilation cross sections from diffuse gamma-ray observations, that turned out to be similarly restrictive as the original one from Ref. [1, 2].

More recently, the PAMELA collaboration published data showing an "anomalous growth" of the cosmic ray positron fraction [7], while the measured antiproton fraction agrees with expectations from simples models [8]. Additionally, new data on the overall electron plus positron spectrum were presented, most notably from the Fermi space telescope $[9,10]$. These data have prompted a plethora of models trying to explain the data by engineering relatively heavy $\left(m_{X} \gtrsim 1 \mathrm{TeV}\right) \mathrm{DM}$ candidates with large annihilation cross sections (or decay rates) and small or vanishing branching ratios (br's) into hadronic final states. In the analysis of these models, the role of radiated $W, Z$ bosons and their phenomenological impact has been generally ignored.

The purpose of this article is to revisit the topic of electroweak bremsstrahlung effects with several goals in mind. First of all, albeit the qualitative conclusions of Refs. [5] and [6] agree, their results differ quantitatively. Here, we repeat this calculations analytically and crosscheck them against numerical results using Madgraph. Second and more important for its recent phenomenological appeal, similar effects also arise when the treelevel final state is a charged lepton $\ell^{+} \ell^{-}$, and therefore we present an analogous calculation for an $e^{+} e^{-}$pair as final state. Third, besides photons, the fragmentation of the emitted $W$ and $Z$ bosons produces also electrons and positrons, protons and antiprotons, as well as neutrinos and antineutrinos (hereafter, simply "neutrinos"). Hence we can use observations of different cosmic species to derive complementary constraints on DM annihilations. Finally, we include in the present analysis new data on the antiproton [8] and positron fraction [7] from the PAMELA satellite as well as on diffuse gamma-rays from the Fermi Gamma-ray Space Telescope [11].

This article is organized as follows: In Sec. II we review first some general considerations about the favoured DM annihilation modes and then our analyt- 
ical calculation of the branching ratio for electroweak bremsstrahlung. In Sec. III we describe the spectra of secondaries found numerically, while Sec. IV is devoted to the constraints from present observations. We conclude in Sec. V.

\section{ANALYTICAL CALCULATION OF B.R.'S INTO $W, Z$.}

\section{A. General considerations}

It is interesting to ask oneself under which circumstances it is possible to produce unsuppressed or even dominant final states consisting of (possibly light or massless) leptons. In the following, for the sake of selfconsistency, we elaborate on some of the considerations reported in the Appendix of [6] and in [12].

On general grounds, the $L$-th partial wave contribution to the annihilation rate of two heavy, non-relativistic particles moving with relative velocity $v$ is suppressed as $v^{2 L}$. The virial velocity in our Galactic halo is only $v \sim 10^{-3}$. Typically, only the $L=0$ partial wave results in observable rates for indirect DM detection today. Admitting $L=1$ final states, terms proportional to $v^{2}$ and terms of similar magnitude that are chirally suppressed as $\propto\left(m_{f} / m_{X}\right)^{2}$, where $m_{f}$ denote the mass of fermions, enter the corresponding annihilation rate: This is notably the case for annihilations via an axial vector current, $J^{P C}=1^{++}$. (We use here the spin-parity notation $J^{P C}$, where $J$ is the total-orbital plus internal-spin quantum number, $P$ the parity and $C$ the charge-parity).

Such a scenario has two classes of phenomenological problems: First, any observable effect now should produce huge effects at early times, when $v$ was larger. Second, multi-body final states, e.g. with e.m. or weak boson radiation emitted from one fermion, proceeds unsuppressed as shown for the case of the axial vector mode in Ref. [13]. In this case, bounds from photon and cosmic ray antimatter searches typically rule-out these models. This provides a general argument to focus on $L=0$ modes only, as we do in the following.

For a spin- $1 / 2$ Majorana fermion candidate, the parity is $P=(-1)^{L+1}$ and the charge parity state $C=$ $(-1)_{\text {orbital }}^{L}(-1)_{\text {spin }}^{S+1}(-1)_{\text {anticomm. }}=(-1)^{L+S}$, where the spin $S=0,1$ for a fermion pair. Since $L=0$ is the only acceptable choice, it follows that $P=-1$. Also, the Majorana nature of the particles implies that it must be even under $C$, hence $S=0$. The only state selected is thus the pseudoscalar $0^{-+}$.

Another class of popular DM candidates are scalars. Again, since we are in the non-relativistic limit, the only unsuppressed annihilation state is the scalar one, with $L=0 \rightarrow P=-1$. Either a fundamental DM scalar or pseudoscalar would lead to a scalar singlet. The state is clearly even under $C$-symmetry. Thus, $J^{P C}=0^{++}$, i.e. only a scalar is allowed. A spin-0 two body particle states is also allowed in case of Dirac and vector particles as
DM candidates, although it is then not the unique choice leading to $L=0$ annihilations [12].

In summary, for the following calculations we can adopt an effective field theory approach and define the initial state as $\mathcal{D}$ coupling with $\mathrm{SM}$ neutrinos $\nu$ as $-\mathrm{i} \lambda \mathcal{D} \bar{\nu} \nu$ or the analogous pseudoscalar coupling $-\mathrm{i} \lambda \mathcal{D} \bar{\nu} \gamma^{5} \nu$. Basically without loss of generality, this is a viable way to obtain on a phenomenological level a treelevel coupling to neutrinos only. Note that the exact flavour-structure of the coupling is irrelevant, since the two large neutrino mixing angles lead after oscillations to a $1: 1: 1$ mixture of flavours. A similar coupling will be adopted for the electron final state case as well.

\section{B. Explicit calculation}

As discussed above, we can replace e.g. the annihilation process $\bar{X} X \rightarrow \bar{\nu} \nu Z$ with the decay $\mathcal{D} \rightarrow \bar{\nu} \nu Z$ choosing $m_{\mathcal{D}}=2 m_{X}$. In particular, this replacement becomes exact, if the annihilation process is mediated by a scalar particle,

$$
R_{Z}=\frac{\sigma(\bar{X} X \rightarrow \bar{\nu} \nu Z)}{\sigma(\bar{X} X \rightarrow \bar{\nu} \nu)}=\frac{\Gamma(\mathcal{D} \rightarrow \bar{\nu} \nu Z)}{\Gamma(\mathcal{D} \rightarrow \bar{\nu} \nu)} .
$$

To be specific, we choose a scalar coupling $\mathcal{L}_{I}=-\lambda \mathcal{D} \bar{\nu} \nu$ between neutrinos and $\mathcal{D}$. The tree-level decay rate of $\mathcal{D}$ in one massless neutrino flavour is then $\Gamma(\mathcal{D} \rightarrow \nu \bar{\nu})=$ $\lambda^{2} m_{\mathcal{D}} /(8 \pi)$. Note that this is a factor two smaller than the decay rate used in Ref. [6]. Also note that in the above expression $\nu$ is implicitly assumed to be a Dirac field, hence equal populations of active left-handed neutrinos $\nu_{L}$ and "sterile" right-handed states $\nu_{R}$ are produced. While there exists no evidence for the existence of $\nu_{R}$ 's up to now, this choice leads to the most conservative bounds: Since the "sterile" right-handed neutrino states $\nu_{R}$ do not participate in electroweak interactions, the br's of the radiative channels would be enhanced restricting the tree-level coupling to $\nu_{L}$ only.

The two diagrams describing the electroweak Bremsstrahlung process and our notation for the momenta are shown in Fig. 1. The corresponding Feynman amplitudes are

$$
\begin{aligned}
& \mathcal{M}_{1}=\bar{u}\left(p_{1}\right) \frac{-\mathrm{i} g}{2 c_{W}} \gamma^{\mu} P_{L} \frac{\mathrm{i}}{\phi_{1}} \varepsilon_{\mu}\left(p_{3}\right)(-\mathrm{i} \lambda) v\left(p_{2}\right), \\
& \mathcal{M}_{2}=\bar{u}\left(p_{1}\right)(-\mathrm{i} \lambda) \frac{\mathrm{i}}{\phi_{2}} \frac{-\mathrm{i} g}{2 c_{W}} \gamma^{\nu} P_{L} \varepsilon_{\nu}\left(p_{3}\right) v\left(p_{2}\right),
\end{aligned}
$$

where we have neglected neutrino masses, $m_{\nu}=0$, as always in the following. Furthermore, $g$ is the weak coupling constant, $c_{W}=\cos \vartheta_{W}$ and $P_{L / R}=\left(1 \pm \gamma^{5}\right) / 2$ are the left/right-chiral projection operators. Using as short-hand notation for the polarization tensor of a massive gauge boson $P_{\mu \nu}=-g_{\mu \nu}+p_{3 \mu} p_{3 \nu} / m_{Z}^{2}$ as well as $K_{i}=\left[(g \lambda) /\left(2 c_{W} q_{i}^{2}\right)\right]^{2}$, we obtain summing over spins

$$
\overline{\mathcal{M}_{1} \mathcal{M}_{1}^{*}}=K_{1} P_{\mu \nu} \operatorname{Tr}\left(\not p_{1} \gamma^{\mu} P_{L} \phi_{1} p_{2} \phi_{1} \gamma^{\nu}\right)=K_{1} P_{\mu \nu} B_{1}^{\mu \nu} .
$$




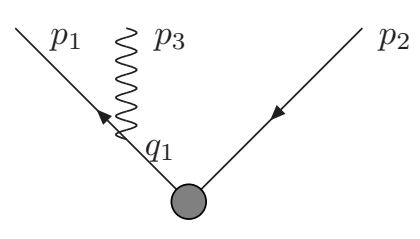

$\mathcal{D}$

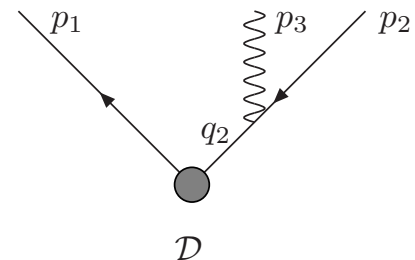

$\mathcal{D}$

FIG. 1: Feynman diagrams for the process $\mathcal{D} \rightarrow \bar{\nu} \nu Z$, left $q_{1}=p-p_{2}$ and right $q_{2}=p-p_{1}$, with $p$ being the $\mathcal{D}$-state four-momentum.

The interference term vanishes for $m_{\nu}=0$ and thus we can set $|\mathcal{M}|^{2}=2\left|\mathcal{M}_{1}\right|^{2}$ in the calculation of the decay rate. Evaluating the trace in the rest-frame of $\mathcal{D}$ gives with $p=\left(m_{\mathcal{D}}, 0\right)$

$$
\begin{aligned}
& \frac{P_{\mu \nu} B_{1}^{\mu \nu}}{q_{1}^{4}}=\frac{2\left(m_{\mathcal{D}}^{2}+m_{Z}^{2}-m_{12}^{2}\right)}{m_{\mathcal{D}}^{2}+m_{Z}^{2}-m_{12}^{2}-m_{23}^{2}} \\
& -\frac{2 m_{\mathcal{D}}^{2} m_{Z}^{2}}{\left(m_{\mathcal{D}}^{2}+m_{Z}^{2}-m_{12}^{2}-m_{23}^{2}\right)^{2}}+\frac{m_{12}^{2}}{m_{Z}^{2}}-2,
\end{aligned}
$$

where we introduced $m_{i j}^{2}=\left(p_{i}+p_{j}\right)^{2}=\left(p-p_{k}\right)^{2}$ [14].

The differential decay rate for a general $1 \rightarrow 3$ decay process,

$$
\mathrm{d} \Gamma=\frac{1}{(2 \pi)^{3}} \frac{1}{32 m_{\mathcal{D}}^{3}}|\overline{\mathcal{M}}|^{2} \mathrm{~d} m_{12}^{2} \mathrm{~d} m_{23}^{2},
$$

can be integrated exactly using the limits [15]

$$
\left(m_{23}^{2}\right)_{\min }^{\max }=\frac{1}{2}\left[m_{\mathcal{D}}^{2} \pm\left(\Delta^{2}-2 \Sigma m_{\mathcal{D}}^{2}+m_{\mathcal{D}}^{4}\right)^{1 / 2}+\Delta\right]
$$

with

$$
\Delta=m_{Z}^{2}-m_{12}^{2} \quad \text { and } \quad \Sigma=m_{Z}^{2}+m_{12}^{2} .
$$

The resulting ratio $R_{Z}$ is

$R_{Z}=\frac{g^{2}}{384 \pi^{2} c_{W}^{2}} y_{Z}\left[1-\frac{9}{y_{Z}}-\frac{9}{y_{Z}^{2}}+\frac{17}{y_{Z}^{3}}+\left(\frac{18}{y_{Z}^{2}}+\frac{6}{y_{Z}^{3}}\right) \ln y_{Z}\right]$

with $y_{Z} \equiv p^{2} / m_{Z}^{2}=4 m_{X}^{2} / m_{Z}^{2}$. The ratio $R_{W}$ follows from $R_{W}\left(y_{W}\right)=2 c_{W}^{2} R_{Z}\left(y_{W}\right)$. Our ratios have the same dependence on $y_{Z, W}$ as those found by the authors of Ref. [6], but are a factor four larger. A factor of two is explained by the difference in the tree-level decay width, while the other, given the agreement of our analytical results with numerical ones presented in the following, is attributed to a missing algebraic factor in the calculation of $[6]$.

For the electron final state case, the ratio $R_{W}$ writes similarly as above, but the different structure of the coupling with $Z$ (involving also $P_{R}$ ) does not lead to a similar simple expression for $R_{Z}$; in particular, interference terms do not vanish in the unitary gauge. The latter contribution has been thus calculated only numerically.

In our calculation of $R_{Z}$ we have included only final state radiation (FSR) neglecting virtual state radiation (VSR) "from the decay vertex" as well as initial state radiation (ISR). The latter two can be only calculated within specific models or assuming a separation of scales such that an effective theory approach can be used. The separation between three classes of bremsstrahlung is gauge-dependent and thus strictly speaking meaningless. However, in cases where one of the three radiation mechanisms dominates and no cancellations are present, this separation is useful. For the electromagnetic case, the relative importance of VSR and FSR is discussed e.g. in Ref. $[13,16,17]$. In some cases, VSR is important since, depending on the exact spin structure of the interaction and the particles involved, the helicity suppression present for certain two body final states can disappear. This is the opposite limit to the one we are discussing here, since we are working within the ansatz of dominant neutrino or electron two-body final states. For our purpose, it is reasonable to conclude that neglecting the (model-dependent) ISR and VSR provides at most a slightly conservative evaluation of the visible final state channels.

Also, it has been shown in [18] that, in addition to the $Z$ and $W$-strahlung processes, loop-induced branching ratios into charged leptons (and, we note, more generally into charged fermions including quarks) are unavoidable in these scenarios. Similarly to the VSR, these effects depend on the UV completion of the model and we do not discuss this further. Typically, one expects these branching ratios to be at the percent level, and thus mainly important for relatively light DM particles, $m_{X} \lesssim m_{W}$. In any case, loop processes as additional source of photons and anti-matter would strengthen the limits derived in the following.

Let us discuss now the behavior of the bremsstrahlung amplitudes in the limit $m_{\mathcal{D}} \gg m_{Z}$. The similar calculation of the three-jet rate in QCD with the emission of a massive gluon leads to $R_{\mathrm{QCD}} \propto \ln ^{2}\left(m_{\mathcal{D}}^{2} / m_{g}^{2}\right)$, and in this process the longitudinal gluon does not contribute as it is coupled to a conserved current. By contrast, the axial vector current is not conserved and it is indeed the longitudinal polarization of the electroweak gauge bosons that is responsible for the quadratic mass dependence, $R_{i} \propto m_{\mathcal{D}}^{2}$ [19]. This difference is also the main reason for the discrepancy between the results of Ref. [5] and [6]. In the former work, the probability for electroweak bremsstrahlung was calculated using the Feynman-'t Hooft gauge and as tree level process $\bar{\nu}^{\prime} \nu^{\prime} \rightarrow \bar{\nu} \nu$. Hence, additionally to the differences ex- 
pected from using a different current and coupling structure mediating the annihilations, the slower rise of the br's (which also holds in SUSY, see below) found in [5] can be attributed to the absence of the longitudinal polarization of the gauge bosons.

Unitarity requires e.g. that the annihilation cross section behaves as $\langle\sigma v\rangle \propto m_{X}^{-2}$. While this bound is respected by the tree-level cross section, the $R_{i}^{2} \propto m_{\mathcal{D}}^{2}$ dependence leads to a violation of perturbative unitarity if an additional $W$ or $Z$ is emitted. The decoupling property of supersymmetry [20] guaranties that such a quadratic term is absent, even in the presence of soft masses $\gg m_{W}$. An explicit calculation in the MSSM show that the ratio of e.g. $\sigma\left(\chi \chi \rightarrow e^{+} e^{-} Z\right) / \sigma(\chi \chi \rightarrow$ $\left.e^{+} e^{-}\right) \sim 0.03$ for a $10 \mathrm{TeV}$ neutralino. Hence the MSSM is an example for a scheme where initial, virtual and final state radiation from all sub-processes in the $s, t$ and $u$ channel is arranged in such a way that their leading contributions cancel. On the other hand, in such a theory quarks and gauge bosons are produced at tree-level in two-body final states, so that the yields of secondaries other than leptons are typically large.

Similarly, the above considerations can be circumvented if the DM does not decay directly into SM particles, rather trough some light state $Y$, so that $W, Z$ in the final state are kinematically inaccessible. These models however introduce other light, metastable degrees of freedom, which violate from the beginning our "effective theory" approach. On the other hand, for a chain $X \bar{X} \rightarrow n Y \rightarrow S M$, provided that $m_{Y} \geq m_{Z}$, one might still apply the above arguments with the new ratios written now as $R_{Z, W}^{\prime}=n R\left(m_{Y}^{2} / m_{Z, W}^{2}\right)$, although the distributions of secondaries will be different, of course.

\section{NUMERICAL RESULTS}

We have calculated the total cross section $\bar{X} X \rightarrow$ $\nu_{e} e^{ \pm} W^{\mp}$ also numerically, using Madgraph [21] and allowing electroweak bremsstrahlung only as FSR. More precisely, we used the MSSM model, choosing $X$ as the lightest neutralino and the scalar higgs $h$ as intermediate particle as well as switching off all diagrams except the one corresponding to FSR. We denote the ratio of the bremsstrahlung and the tree-level processes again as $R_{W}$,

$R_{W}=\frac{\sigma_{3}}{\sigma_{2}}=\frac{\sigma\left(\bar{X} X \rightarrow \nu_{e} e^{+} W^{-}\right)+\sigma\left(\bar{X} X \rightarrow \bar{\nu}_{e} e^{-} W^{+}\right)}{\sigma\left(\bar{X} X \rightarrow \bar{\nu}_{e} \nu_{e}\right)}$.

In Fig. 2, we compare our analytical result for $R_{W}$ with the numerical results obtained with Madgraph, finding excellent agreement.

Note that the perturbative results for the bremsstrahlung cross sections become unreliable already at $m_{X} \gtrsim 1 \mathrm{TeV}$. Above this energy, processes with $n>3$ (treating $W, Z$ as stable) particles in the final state become important and an electroweak cascade

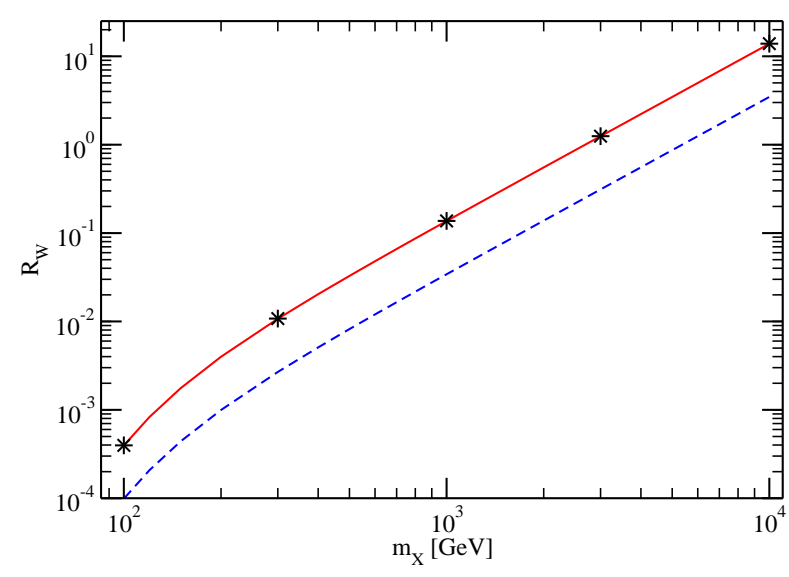

FIG. 2: Our analytical result for the ratio $R_{W}$ (solid line), compared to $R_{W}$ from ref. [6] (dashed line) and the numerical results from Madgraph (points).

develops [4]. On the other hand, each individual subprocess is suppressed by a Sudakov factor compared to the perturbative result, avoiding a "blowing-up" of the total annihilation cross section.

In the next step, we feed the events generated by Madgraph into HERWIG $++[22]$ and generate the energy spectra of $e^{ \pm}, \nu_{i}, \bar{\nu}_{i}, p$, and $\bar{p}$ produced as secondaries in $W$ and $Z$ decays. The obtained energy spectrum $\mathrm{d} N / \mathrm{d} x$ are normalized to the total cross section, $\sigma_{\text {tot }}=\sigma_{2}+\sigma_{3}$,

$$
\frac{\mathrm{d} N_{i}}{\mathrm{~d} x}=\frac{f_{i}}{\sigma_{\mathrm{tot}}} \frac{\mathrm{d} \sigma(X X \rightarrow i+\text { all })}{\mathrm{d} x}
$$

where $f_{i}$ is the multiplicity to produce particles of type $i$ with energy $E=x m_{X}$. Therefore the evolution of $\mathrm{d} N / \mathrm{d} x$ with energy becomes much slower for $m_{X} \sim$ few $\mathrm{TeV}$, when $\sigma_{\mathrm{tot}} \approx \sigma_{3} \gg \sigma_{2}$. Since the shape of the fragmentation function $\mathrm{d} N_{i} / \mathrm{d} x$ changes only logarithmically, the omission of $2 \rightarrow n>3$ processes has only a minor impact on $\mathrm{d} N_{i} / \mathrm{d} x$. Moreover, the chosen normalization gives the correct number of secondaries $N_{i}$ per annihilation also for $\sigma_{3} \gg \sigma_{2}$. The resulting fragmentation functions $\mathrm{d} N / \mathrm{d} x$ as function of $x$ are shown for $m_{X}=300 \mathrm{GeV}$ and $m_{X}=3 \mathrm{TeV}$ in Fig. 3. Note that i) there is an additional contribution $\delta(1-x) \sigma_{2} / \sigma_{\text {tot }}$ which is not reported for clarity and ii) only half of the neutrinos emitted at tree-level are "active" ones, $\nu_{L}$.

In the same way as described above for tree-level annihilations into neutrinos, we have calculated also the total cross sections of $\bar{X} X \rightarrow e^{+} e^{-}$and $\bar{X} X \rightarrow e^{+} e^{-} Z$, $\bar{X} X \rightarrow \nu_{e} e^{ \pm} W^{\mp}$. Additionally, we added analytically photons from external electromagnetic bremsstrahlung,

$$
\frac{\mathrm{d} N_{\gamma}}{\mathrm{d} x}=\frac{\alpha}{\pi} P_{\mathrm{ff}}(x) \ln \frac{s(1-x)}{m_{e}^{2}}
$$



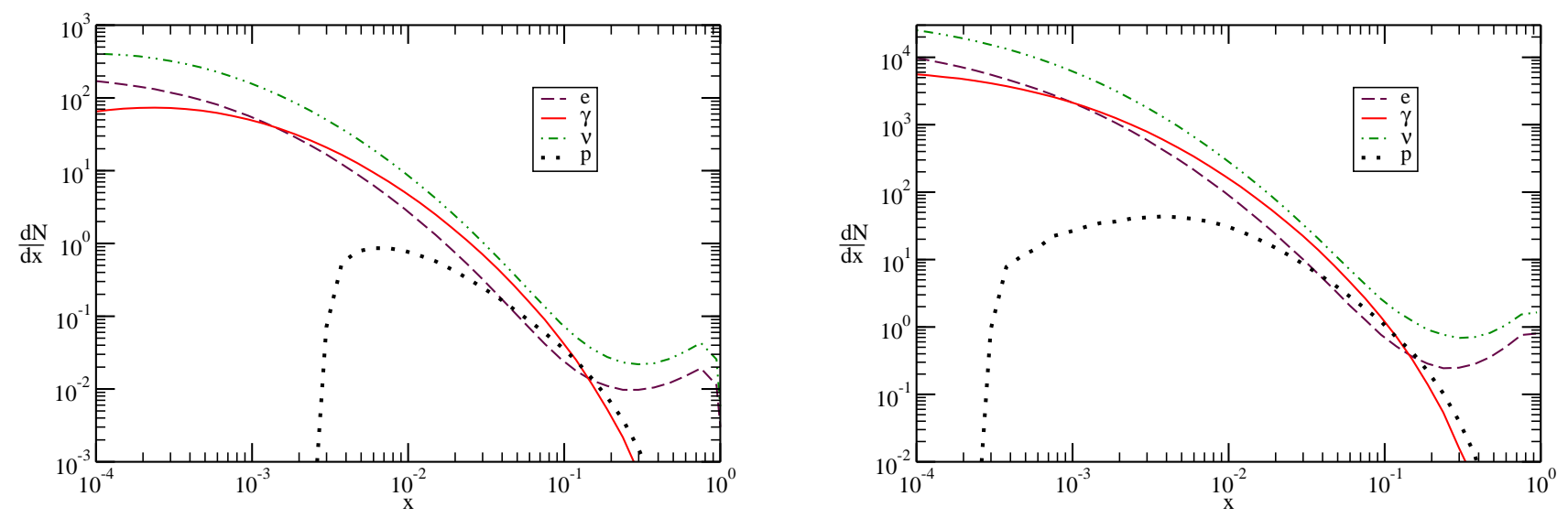

FIG. 3: The fragmentation spectra $\mathrm{d} N / \mathrm{d} x$ of electrons (long-dashed maroon), photons (solid red), neutrinos (dot-dot-dashed green), and protons (dotted black) in "only neutrinos" tree level annihilations for $m_{X}=300 \mathrm{GeV}$ (left) and $m_{X}=3 \mathrm{TeV}$ (right). The monochromatic neutrino "spike" at $x=1$ is not shown. Fermion labels indicate the sum of matter and antimatter.

with the usual fermion-fermion-boson splitting function

$$
P_{\mathrm{ff}}(x)=\frac{1+(1-x)^{2}}{x} .
$$

The resulting fragmentation functions $\mathrm{d} N_{i} / \mathrm{d} x$ are shown for $m_{X}=300 \mathrm{GeV}$ and $m_{X}=3 \mathrm{TeV}$ in Fig. 4 . Note that the relative importance of protons is largest for $x \sim 0.1$ where it is comparable with the secondary electron flux. Also, bremsstrahlung photons (absent for the neutrino case) provide the dominant yield only for subTeV DM masses, while for heavy DM particles, apart for the region $x \gtrsim 0.1$ and the very small $x$, the photons from $W, Z$ fragmentation dominate. As above, there is an additional contribution to the $e^{-}+e^{+}$case of $2 \delta(1-x) \sigma_{2} / \sigma_{\text {tot }}$ which is not reported for clarity, but of course included in the constraints derived below. Note also that, in contrast to the neutrino case, both helicity states emitted at tree-level contribute to the observable electron spectrum.

\section{CONSTRAINTS}

In this section we provide a first application of the calculations reported above, deriving bounds on $\langle\sigma v\rangle$ vs. $m_{X}$ from measurements of the diffuse gamma ray flux, the antiproton fraction, the electron plus positron flux, the positron fraction and the limits on Galactic neutrino fluxes. We shall adopt a simple prescription, namely we consider a model as excluded when the DM contribution to the signal alone exceeds the maximal flux allowed by the data at one sigma. This is likely over-conservative since it is known that other more mundane astrophysical sources contribute to or even dominate the fluxes.
For easing the comparison with other papers, we adopt for the smooth DM mass density $\rho_{\mathrm{sm}}$ a fiducial NavarroFrenk-White profile [23]

$$
\rho_{\mathrm{sm}}(r)=\rho_{\odot}\left(\frac{r_{\odot}}{r}\right)\left(\frac{r_{\odot}+a}{r+a}\right)^{2}
$$

where $a=20 \mathrm{kpc}$ is the characteristic scale below which the profile scales as $r^{-1}$. Following the new determination in [24], we choose $\rho_{\odot}=0.39 \mathrm{GeV} / \mathrm{cm}^{3}$ as the DM density at the solar distance from the (Galactic center) GC; the latter is estimated to be $r_{\odot} \approx 8.33 \mathrm{kpc}$ [25]. Since we shall refer to high-latitude fluxes (for the photon case) or diffuse signals (for the charged particles cases) the exact DM profile towards the GC is not essential. For neutrinos, we shall limit this dependence adopting a quite large cone size (see below).

Note that additional bounds can be derived by focusing on more specific location (as the GC) and/or by looking at other channels produced as secondaries of leptonic energy losses (inverse Compton, synchrotron radiation,... ). It is beyond the scope of this paper to provide an exhaustive account of indirect bounds. Here we only note that most of them depend more on the properties of the Galactic medium, and that further constraints can only strengthen the results presented here. 

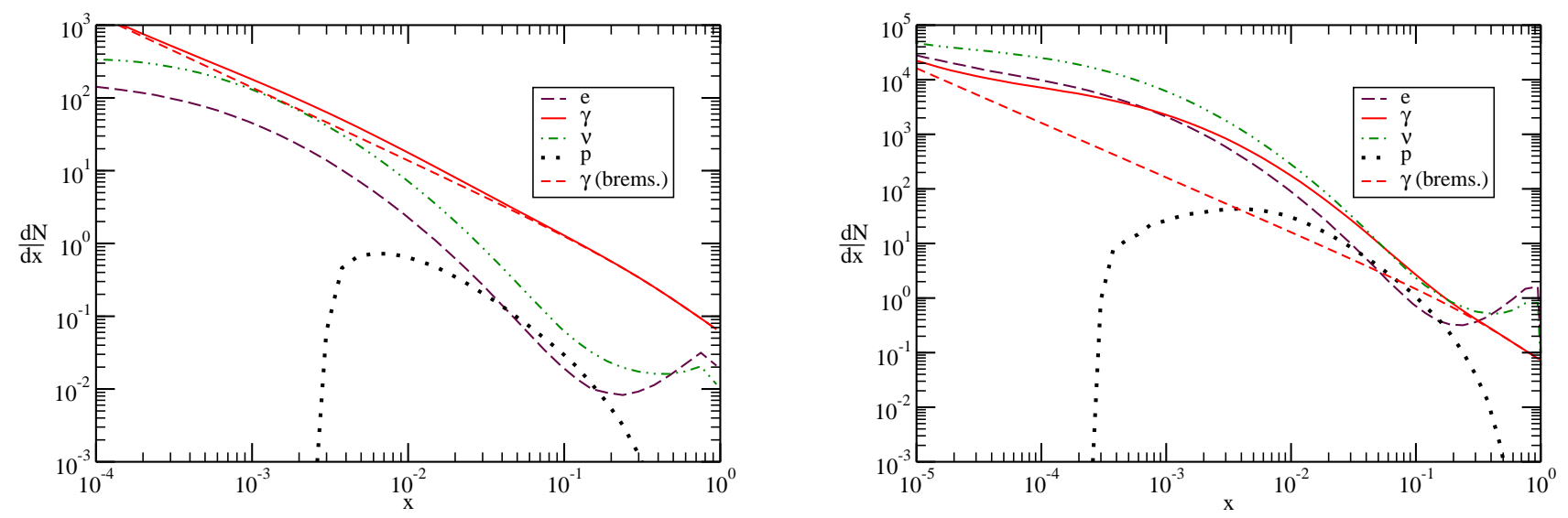

FIG. 4: The fragmentation spectra $\mathrm{d} N / \mathrm{d} x$ of electrons (long-dashed maroon), photons (solid red), neutrinos (dot-dot-dashed green), and protons (dotted black) in "only electron" annihilations for $m_{X}=300 \mathrm{GeV}$ (left) and $m_{X}=3 \mathrm{TeV}$ (right). Also shown is the gamma-ray yield from bremsstrahlung only (short-dashed red). The monochromatic electron "spike" at $x=1$ is not shown. Fermion labels indicate the sum of matter and antimatter.

\section{A. Gamma-rays}

The differential flux of photons from (self-conjugated) dark matter annihilations is

$$
\Phi_{\gamma}(E, \psi)=\frac{\mathrm{d} N_{\gamma}}{\mathrm{d} E} \frac{\langle\sigma v\rangle \rho_{\odot}^{2}}{8 \pi m_{X}^{2}} \int_{\text {l.o.s. }} \mathrm{d} s\left(\frac{\rho_{\mathrm{sm}}[r(s, \psi)]}{\rho_{\odot}}\right)^{2},
$$

where $r(s, \psi)=\left(r_{\odot}^{2}+s^{2}-2 r_{\odot} s \cos \psi\right)^{1 / 2}, \psi$ is the angle between the direction in the sky and the GC and $s$ the distance from the Sun along the line-of-sight (l.o.s.). In terms of the galactic latitude $b$ and longitude $l$, one has $\cos \psi=\cos b \cos l$. Particle physics enters via the DM mass $m_{X}$, the annihilation cross section $\langle\sigma v\rangle$, and the photon differential energy spectrum $\mathrm{d} N_{\gamma} / \mathrm{d} E$ per annihilation. Since here we are focusing on the galactic diffuse emission rather than that from the GC, the residual uncertainties which are introduced through the choice of the DM profile are negligible for our discussion. We shall compare the calculated flux with the high-latitude residual "isotropic" emission whose preliminary data have been presented by the Fermi-LAT collaboration [11], whose upper limit at $\sim 1 \sigma$ in the range $0.1-50 \mathrm{GeV}$ can be roughly represented by $E^{2} \mathrm{~d} N / \mathrm{d} E \simeq$ $1.5 \times 10^{-2}(E / 0.1 \mathrm{GeV})^{-0.45} \mathrm{GeV} \mathrm{m}^{-2} \mathrm{~s}^{-1} \mathrm{sr}^{-1}$.

\section{B. Antiprotons}

Accounting for diffusion, the flux of antiprotons at Earth is isotropic to a high accuracy. By neglecting energy losses and the so-called "tertiary" component (which is mostly relevant at low-energies) the flux can be written similarly to Eq. (15) as

$$
\Phi_{\bar{p}}(E)=\frac{\mathrm{d} N_{\bar{p}}}{\mathrm{~d} E} \frac{\langle\sigma v\rangle \rho_{\odot}^{2}}{8 \pi m_{X}^{2}} \mathcal{F}_{\bar{p}}(E),
$$

where $\mathrm{d} N_{\bar{p}} / \mathrm{d} E$ is now the antiproton injection spectrum per annihilation, while the integral along the line of sight is effectively replaced by a function $\mathcal{F}_{\bar{p}}(E)$ which encodes the dependence of the flux from astrophysical parameters (see [26] for a derivation of the above formula and an explicit expression of $\mathcal{F}_{\bar{p}}$ ). For the present illustrative purposes, we adopt the fit of this function calculated numerically in [27], for the reference NFW model and the "intermediate" choice of propagation parameters (see [27] for details).

In order to compare with the $\bar{p} / p$ ratio provided in [8], we normalize the above calculated flux to the observed proton flux which we take from [28] to be

$$
\Phi_{p}^{\mathrm{obs}}(E)=1.4 \times 10^{4}(E / \mathrm{GeV})^{-2.7} \mathrm{GeV}^{-1} \mathrm{~m}^{-2} \mathrm{~s}^{-1} \mathrm{sr}^{-1},
$$

where we accounted for a proton contribution of $79 \%$ in the overall cosmic ray flux at the energies of interest.

\section{Electrons and Positrons}

Compared to the above case, the main difference concerning the calculation of the flux of $e \equiv\left(e^{-}+e^{+}\right)$at the Earth starting from the injected parameters is due to the relevance of energy losses. A semi-analytical form can be derived $[29,30]$, which yields

$$
\Phi_{\mathrm{e}}(E)=\frac{\mathrm{d} N_{\mathrm{e}}}{\mathrm{d} E} \frac{\langle\sigma v\rangle \rho_{\odot}^{2}}{8 \pi m_{X}^{2}} \mathcal{F}_{\mathrm{e}}(E),
$$


with

$$
\mathcal{F}_{\mathrm{e}}(E)=\frac{1}{\frac{\mathrm{d} N_{\mathrm{e}}}{\mathrm{d} E}(E) b(E)} \int_{E}^{m_{X}} \mathrm{~d} \mathcal{E} \frac{\mathrm{d} N_{\mathrm{e}}}{\mathrm{d} E}(\mathcal{E}) h_{\mathrm{e}}(\mathcal{E}) .
$$

In the above equation, $b(E) \approx 10^{-16}(E / \mathrm{GeV})^{2} \mathrm{GeV} \mathrm{s}^{-1}$ encodes energy losses and the function $h_{\mathrm{e}}(E)$ depends on halo parameters as well as propagation ones. As for the case of antiprotons, we adopt the fit for this function provided in [27] for the NFW case and intermediate propagation parameters. Note that the positron flux amounts to half of the flux reported above.

From the overall electron plus positron spectrum we know from the Fermi measurement that $[9,10]$

$$
\begin{aligned}
\Phi_{\mathrm{e}}^{\mathrm{obs}}(E) \equiv \Phi_{\mathrm{e}^{+}+\mathrm{e}^{-}}^{\mathrm{obs}}(E)= \\
(175.40 \pm 6.09)\left(\frac{E}{\mathrm{GeV}}\right)^{-3.045 \pm 0.008} \frac{1}{\mathrm{GeV} \mathrm{m}^{2} \mathrm{~s} \mathrm{sr}}
\end{aligned}
$$

represents a good fit of the data between $\sim 20 \mathrm{GeV}$ and $1 \mathrm{TeV}$. We shall then require that: i) $\Phi_{\mathrm{e}} /\left(2 \Phi_{\mathrm{e}}^{\mathrm{obs}}\right) \leq$ $f_{\mathrm{e}^{+}}^{\mathrm{up}}(E)$, where $f_{\mathrm{e}^{+}}^{\mathrm{up}}(E)$ is the upper value of the datapoints presented by PAMELA at energies between $\sim 20$ and $\sim 100 \mathrm{GeV}$. ii) That $\Phi_{\mathrm{e}} \lesssim \Phi_{\mathrm{e}}^{\text {obs, up }}$, where $\Phi_{\mathrm{e}}^{\mathrm{obs} \text {, up }}$ is the flux obtained for the upper value of the flux normalization and the hardest spectrum reported in Eq. (20).

\section{Neutrinos}

For neutrinos, we use the Super-Kamiokande bound [31] requiring that the induced flux of muon tracks from a cone of half-width $30^{\circ}$ around the GC is below $1.6 \times 10^{-14} / \mathrm{s}$ (we specifically use the slightly conservative prescription to use only events induced by neutrinos above $10 \mathrm{GeV}$ energy). Also, we assume a $1: 1: 1$ mixture of flavours, which holds within factors of order unity independently of the production flavour ratios due to mixing. Finally, note that the width of the cone around the Galactic Center is such that the dependence of the flux on the exact DM profile is marginal for our purposes (within a factor 2 at most, see $[2])$.

\section{E. Results}

In Fig. 5 we summarize our bounds. Not surprisingly, for the case of an electron final state at tree level (top panel), the bounds from electron-related observables dominate at low energies. In particular, around $m_{X} \sim$ $100 \mathrm{GeV}$, the positron fraction is saturated by a cross section less than one order of magnitude larger than the fiducial value for a thermal relic, $\langle\sigma v\rangle \simeq 3 \times 10^{-26} \mathrm{~cm}^{3} / \mathrm{s}$. Equivalently, we expect a DM contribution above the $10 \%$ level in some bins of the PAMELA data, even in absence of astrophysical boost factors due to a clumpy DM distribution. Note also that for the same mass range
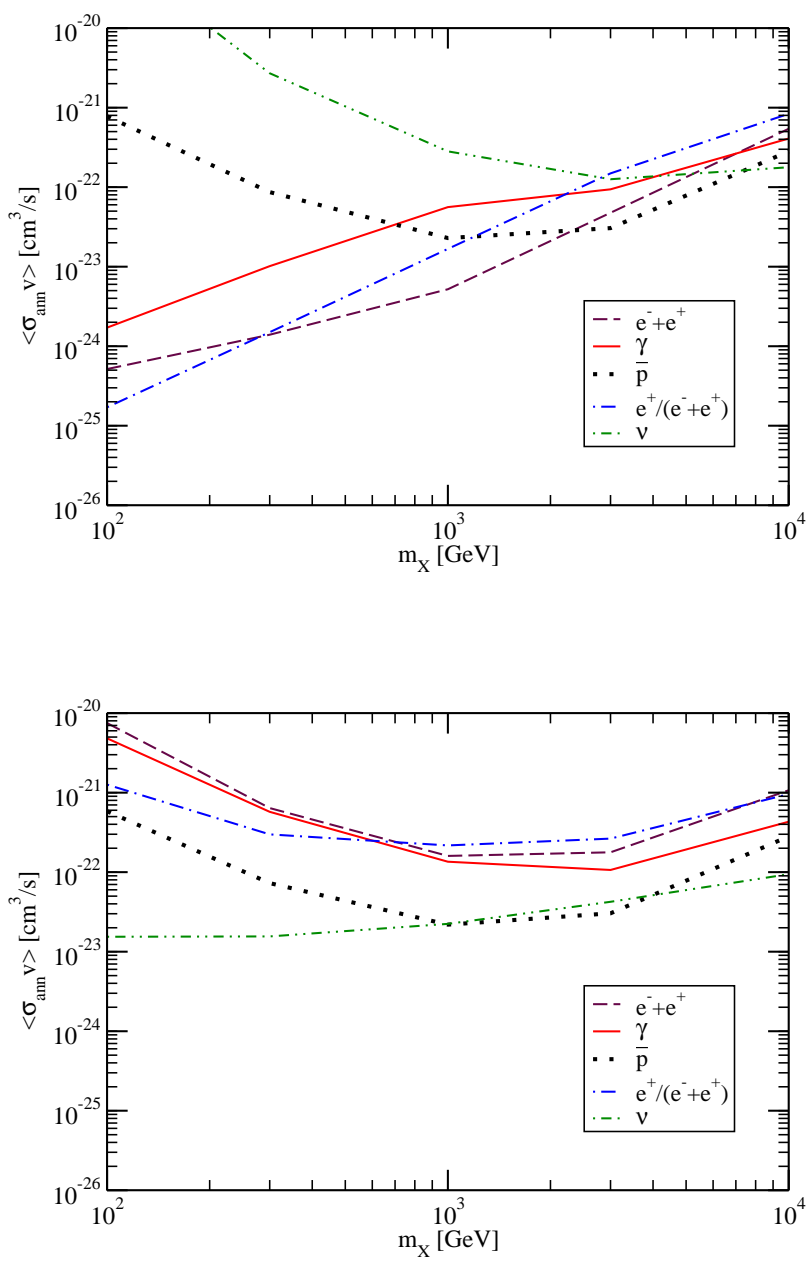

FIG. 5: In the top (bottom) panel, we report the exclusion plot in the $\langle\sigma v\rangle$ vs. $m_{X}$ plane for the case of electron (neutrino) final states at tree level. Regions above the different curves are excluded by the labelled CR observables as described in the text.

the diffuse gamma-ray bounds fall, despite the $\alpha / \pi$ suppression, within two orders of magnitude of the fiducial value, confirming the promising role of this channel for detection in case of "ordinary" final state br's and of more targeted searches (see e.g. [32] and refs. therein). Above $m_{X} \sim \mathrm{TeV}$, the limits derived from different channels have roughly the same strength. Hence saturating the electron flux by DM annihilations will lead to tensions with the antiproton bound, and eventually with the gamma-ray and neutrino bounds too.

For the neutrino final state the best limit comes from neutrino observations, but for the region of a few $\mathrm{TeV}$ where the antipronton fraction provides a better constraint. Also, in the same range the other channels lead to constraints looser by less than one order of magnitude, with the gamma-ray being the most competitive one. 
In deriving the above bounds, we assumed conservatively that the products of the electron energy losses are not observed otherwise, while it is known that they can lead to potentially interesting signatures in "softer" gamma-rays via inverse Compton scattering, for example. Also, we ignored gamma-ray observations closer to the GC, while it is estimated that gamma-ray constraints at intermediate galactic latitudes or towards the GC are stronger than the ones presented here (see e.g. [33-35].) Finally, enforcing the HESS constraints [38] on the combined $e^{-}+e^{+}+\gamma$ flux at energies $E \approx 0.6-4 \mathrm{TeV}$ might produce - depending on the propagation parametersbounds a factor of a few more stringent than the ones shown. Even with these caveats in mind, however, it it interesting to note that multi- $\mathrm{TeV}$ scale particles annihilating into neutrinos at tree-level might turn out to be similarly or even more constrained than particles annihilating into electrons.

The comparison with the electron case shows that even for the largest masses the tree-level, monochromatic neutrinos provide the leading constraint. This is essentially due to two facts: i) The cross section for muon production and the muon range continue to increase also for $s \gg m_{X}^{2}$, partially compensating the $m_{X}^{-2}$ suppression in the differential flux. ii) The lack of energy losses for neutrinos, which is instead a key factor for very energetic electrons.

On general grounds, it appears safe to conclude that if a DM candidates in the few $\mathrm{TeV}$ mass range contributes a significant fraction of the CR electron/positron flux, then a non-negligible antiproton flux is produced as well that is close to the current bound. Especially in the light of the more refined measurements expected in the next years with AMS-02 [36], this is an important signature to keep in mind. Further, for the same class of candidates one expects measurable signatures in both the gammaray and the neutrino flux, with interesting observational perspectives from the direction of the inner Galaxy.

\section{CONCLUSIONS}

We discussed the role of electroweak bremsstrahlung for indirect dark matter signatures, which has been typically neglected in phenomenological studies heretofore. Our approach was to calculate the branching ratios and the fragmentation spectra $\mathrm{d} N_{i} / \mathrm{d} x$ of secondaries considering electroweak radiation only from the final state.
Therefore, our results are applicable mainly to models tailored to produce only leptons as final state and with $\mathrm{DM}$ mass in the $\mathrm{TeV}$ range. In particular, our analysis applies to several models trying to match features in $e^{+} e^{-} \mathrm{CR}$ data with $\mathrm{TeV}$-scale $\mathrm{DM}$, but e.g. not to the benchmark case of neutralino annihilations in the MSSM (where final states containing hadrons or gauge bosons are anyway allowed at tree-level).

An important phenomenological consequence of electroweak bremsstrahlung is its impact on the predicted photon and electron/positron spectra. Secondaries from $W$ and $Z$ decays can dominate in a certain $x$ range the spectra, leading to changes in the normalization and the shape of the secondary spectra. Thus it is mandatory to assess the importance of these "corrections" in a specific model under consideration, before one attempts to fit cosmic ray or photon data. Models viable at tree level may be ruled out by the more realistic spectrum or, vice versa, large boost factors required might be moderately loosened.

On more general grounds, at least in cases where no new light particles are introduced in the spectrum, it appears that $\mathrm{DM}$ candidates in the few $\mathrm{TeV}$ mass range contributing a significant fraction of the CR electron/positron flux should lead also to a non-negligible antiproton flux, close to the current bound. Additionally, for the same class of candidates we expect measurable signatures in both the gamma-ray and the neutrino flux. These "multimessenger" signatures are not surprising and have often been implicitly assumed in past phenomenological works on indirect DM detection. However, it is interesting that the conclusion holds virtually unchanged also for heavy DM candidates engineered to produce only leptons as tree level final states.

\section{Acknowledgments}

We are grateful to J. Alwall and T. Stelzer for advice on Madgraph, and especially to David Grellscheid for help using HERWIG ++ .

Note added: During the preparation of this work, some of the considerations developed here have been mentioned (in a different context) in the model presented in Ref. [37].
[1] J. F. Beacom, N. F. Bell and G. D. Mack, Phys. Rev. Lett. 99, 231301 (2007) [arXiv:astro-ph/0608090].

[2] H. Yuksel, S. Horiuchi, J. F. Beacom and S. Ando, Phys. Rev. D 76, 123506 (2007) [arXiv:0707.0196 [astro-ph]].

[3] S. Palomares-Ruiz, Phys. Lett. B 665, 50 (2008) [arXiv:0712.1937 [astro-ph]].

[4] V. Berezinsky, M. Kachelrieß and S. Ostapchenko, Phys.
Rev. Lett. 89, 171802 (2002) [arXiv:hep-ph/0205218].

[5] M. Kachelrieß and P. D. Serpico, Phys. Rev. D 76, 063516 (2007) [arXiv:0707.0209 [hep-ph]].

[6] N. F. Bell, J. B. Dent, T. D. Jacques and T. J. Weiler, Phys. Rev. D 78, 083540 (2008) [arXiv:0805.3423 [hep$\mathrm{ph}]$.

[7] O. Adriani et al., Nature 458, 607 (2009). 
[arXiv:0810.4995].

[8] O. Adriani et al., Phys. Rev. Lett. 102, 051101 (2009) [arXiv:0810.4994 [astro-ph]].

[9] A. A. Abdo et al. [The Fermi LAT Collaboration], Phys. Rev. Lett. 102, 181101 (2009) [arXiv:0905.0025 [astroph.HE]].

[10] D. Grasso et al. [FERMI-LAT Collaboration], Astropart. Phys. 32, 140 (2009) [arXiv:0905.0636 [astro-ph.HE]].

[11] Markus Ackermann, talk at $\mathrm{TeV}$ Particle Astrophysics 2009, slides available at http://www-conf.slac. stanford.edu/tevpa09/Talks.asp

[12] M. Cirelli, M. Kadastik, M. Raidal and A. Strumia, Nucl. Phys. B 813, 1 (2009) [arXiv:0809.2409 [hep-ph]].

[13] L. Bergstrom, Phys. Lett. B 225, 372 (1989). R. Flores, K. A. Olive and S. Rudaz, Phys. Lett. B 232, 377 (1989).

[14] We used for the evaluation of the trace the package of T. H. West, Comput. Phys. Commun. 77, 286 (1993).

[15] C. Amsler et al. [PDG], PhysLett. B667, 1 (2008), sec. "Kinematics".

[16] T. Bringmann, L. Bergstrom and J. Edsjo, JHEP 0801, 049 (2008) [arXiv:0710.3169 [hep-ph]].

[17] V. Barger, Y. Gao, W. Y. Keung and D. Marfatia, Phys. Rev. D 80, 063537 (2009) [arXiv:0906.3009 [hep-ph]].

[18] J. B. Dent, R. J. Scherrer and T. J. Weiler, Phys. Rev. D 78, 063509 (2008) [arXiv:0806.0370 [astro-ph]].

[19] For a possible connection to "anomalous" Sudakov form factors see M. Ciafaloni, P. Ciafaloni and D. Comelli, arXiv:0909.1657 [hep-ph].

[20] A. Dobado, M. J. Herrero and S. Penaranda, arXiv:hep-ph/9806488, Eur. Phys. J. C 12, 673 (2000) [hep-ph/9903211]; V. Berezinsky, M. Kachelrieß and M. A. Solberg, Phys. Rev. D 78, 123535 (2008) [arXiv:0810.3012 [hep-ph]].

[21] J. Alwall et al., JHEP 0709, 028 (2007) [arXiv:0706.2334 [hep-ph]]; http://madgraph.hep.uiuc.edu/

[22] M. Bahr et al., Eur. Phys. J. C 58, 639 (2008)
[arXiv:0803.0883 [hep-ph]]; http://projects. hepforge.org/herwig/

[23] J. F. Navarro, C. S. Frenk and S. D. M. White, Astrophys. J. 462, 563 (1996) [astro-ph/9508025].

[24] R. Catena and P. Ullio, arXiv:0907.0018 [astro-ph.CO].

[25] S. Gillessen, F. Eisenhauer, S. Trippe, T. Alexander, R. Genzel, F. Martins and T. Ott, Astrophys. J. 692, 1075 (2009) [arXiv:0810.4674 [astro-ph]].

[26] F. Donato, N. Fornengo, D. Maurin and P. Salati, Phys. Rev. D 69, 063501 (2004) [arXiv:astro-ph/0306207].

[27] M. Cirelli, R. Franceschini and A. Strumia, Nucl. Phys. B 800, 204 (2008) [arXiv:0802.3378 [hep-ph]].

[28] C. Amsler et al. [PDG], PhysLett. B667, 1 (2008), sec. "Cosmic Rays".

[29] J. Hisano, S. Matsumoto, O. Saito and M. Senami, Phys. Rev. D 73, 055004 (2006) [arXiv:hep-ph/0511118].

[30] T. Delahaye, R. Lineros, F. Donato, N. Fornengo and P. Salati, Phys. Rev. D 77, 063527 (2008) [arXiv:0712.2312 [astro-ph]].

[31] S. Desai et al. [Super-Kamiokande Collaboration], Phys. Rev. D 70, 083523 (2004) [Erratum-ibid. D 70, 109901 (2004)] [arXiv:hep-ex/0404025].

[32] P. D. Serpico and D. Hooper, New J. Phys. 11, 105010 (2009) [arXiv:0902.2539 [hep-ph]].

[33] S. Dodelson, D. Hooper and P. D. Serpico, Phys. Rev. D 77, 063512 (2008) [arXiv:0711.4621 [astro-ph]].

[34] P. D. Serpico and G. Zaharijas, Astropart. Phys. 29, 380 (2008) [arXiv:0802.3245 [astro-ph]].

[35] E. A. Baltz et al., JCAP 0807, 013 (2008) [arXiv:0806.2911 [astro-ph]].

[36] http://ams.cern.ch/

[37] A. Falkowski, J. Juknevich and J. Shelton, arXiv:0908.1790 [hep-ph].

[38] F. Aharonian et al. [H.E.S.S. Collaboration], Phys. Rev. Lett. 101, 261104 (2008) [arXiv:0811.3894 [astro-ph]]. 